\title{
TTR
}

Traduction, terminologie, re?daction

\section{Maurice Pergnier, Du sémantique au poétique avec Baudelaire, Cocteau, Magritte (Préface de Denis Slatka). Paris-Montréal, L'Harmattan, 1997, 127 p.}

\section{Claude Tatilon}

Volume 12, numéro 1, 1er semestre 1999

Poésie, cognition, traduction I

Poetry, Cognition, Translation I

URI : https://id.erudit.org/iderudit/037361ar

DOI : https://doi.org/10.7202/037361ar

Aller au sommaire du numéro

Éditeur(s)

Association canadienne de traductologie

ISSN

0835-8443 (imprimé)

1708-2188 (numérique)

Découvrir la revue

Citer ce compte rendu

Tatilon, C. (1999). Compte rendu de [Maurice Pergnier, Du sémantique au poétique avec Baudelaire, Cocteau, Magritte (Préface de Denis Slatka). Paris-Montréal, L'Harmattan, 1997, 127 p.] TTR, 12(1), 196-200.

https://doi.org/10.7202/037361ar d'utilisation que vous pouvez consulter en ligne.

https://apropos.erudit.org/fr/usagers/politique-dutilisation/ 
Maurice Pergnier, Du sémantique au poétique avec Baudelaire, Cocteau, Magritte (Préface de Denis Slatka). Paris-Montréal, L'Harmattan, 1997, 127 p.

La lecture ordinaire est acte de consommation d'un sens manifeste. Pour qui est plongé dans les nouvelles de son journal, le langage a la transparence d'une vitre : son regard le traverse sans le remarquer pour ne s'intéresser qu'au monde aperçu derrière. Le lecteur d'un texte poétique sait, lui, que cette fois le spectacle se trouve sur la vitre. Opaque, celle-ci ne laisse alors passer qu'un reflet du monde extérieur et donne surtout à voir ses propres craquelures. Cette métaphore a souvent été utilisée - ou d'autres analogues comme, chez Paul Valéry, 
celle de la marche et de la danse - pour opposer la fonction utilitaire d'une prose courante à la fonction poétique d'une écriture d'artiste. Maurice Pergnier la reprend, dans une formulation qui ne manque pas de vigueur : " Dans la communication ordinaire, les mots visent à la transparence. Dans la poésie, au contraire, ils sont translucides. La prose les veut vitres, la poésie vitraux » (p. 81). Tout son livre traite de cet « art du vitrail ».

Après Jakobson et beaucoup d'autres (par exemple, Mounin, Granger et Riffaterre, pour avouer mes préférences), Pergnier tente ici d'élucider le mystère de l'usage poétique du langage, cet usage particulier qui réussit à faire exprimer aux textes beaucoup plus que le sens manifeste qu'ils transmettent ordinairement. Et, à partir d'une position aujourd'hui largement admise, à savoir que le surcroît de sens constituerait l'essence même du poétique, il prône une lecture syncrétique embrassant à la fois toutes les suggestions légitimes d'une œuvre. « Le poème, déclare-t-il à propos d'un texte de Jean Cocteau, n'est pas un rébus. Son sens réside dans la pluralité de lectures simultanées " (p. 62). Ou encore, à propos du même texte : " Ce qui fait le poème, c'est justement que [ses] strates de lecture se complètent, autant qu'elles divergent pour créer un effet de lecture à multiples facettes. Le poème n'est nulle part ailleurs que dans les réfractions de sens à travers l'homonymie " (p. 70).

Faisant, dans son deuxième chapitre intitulé « Le signe équivoque ", une claire distinction entre la polysémie, phénomène inhérent à la langue (un même signe linguistique étant généralement investi d'une pluralité de signifiés possibles), et l'ambiguiité, phénomène de discours, il " scrute " quatre objets esthétiques (trois poèmes et un tableau) et découvre, dans l'utilisation extensive que leurs auteurs font de la polysémie alors étendue à l'énoncé, « une figure non répertoriée, qui ne se confond pas avec le "double-entendre» caractérisé dont on a pu relever des exemples aussi bien dans de nombreux poèmes que dans des textes en prose " (p. 38). Par son étymologie (lat. ambo, " les deux ensemble »), ambiguîté est un terme malheureux, puisque Pergnier lui donne, conformément à une acception courante il est vrai, le sens de polysémie (plusieurs sens). C'est pourquoi il insiste pour clairement distinguer l'ambiguité ainsi entendue du double-entendre - à propos de ce dernier, qu'on songe à certains slogans publicitaires du genre de celui-ci (concernant un 
parapluie) : Il vous plaira avant même qu'il ait plu, ou à certaines facéties d'auteurs, de Victor Hugo par exemple : Ce repas me tracasse et même me harcèle; J'ai mangé du cheval et je songe à la selle (dont je ne garantis pas la ponctuation). Lorsque, par un minutieux travail d'écriture, la polysémie, qui appartient à la langue, est étendue au discours, elle produit un agrandissement « poétique » du sens. Pergnier précise, dans ce même chapitre : "Lorsque le langage poétique construit des trames signifiantes qui permettent à plusieurs [...] acceptions de se présenter à l'esprit simultanément, il abolit en même temps l'obligation de choisir qui prévaut dans l'utilisation pragmatique du langage, pour créer un sens qui transcende celui de chacune des acceptions tout en remontant aux racines de leur apparentement " (p. 51). Plus loin, il avance un "principe de solidarité des lectures superposées » (p. 100) et écrit encore, à propos d'un second poème de Cocteau : " Aucune de ces interprétations n'est contredite par le texte. Mais ce dernier n'existe comme poème qu'en tant qu'il "pétrifie" en un même être de langage autonome ces différentes virtualités de compréhension qui tantôt se complètent et tantôt se contredisent " (p. 103). En outre, lorsqu'il affirme que la poésie " est — avant toute chose - une façon de faire dire au langage ce qu'il n'a jamais dit auparavant $»$ (p. 107), Pergnier parle alors exactement comme Georges Mounin. Mais cette frappante similitude de voix ne doit pas cacher la profonde différence qui les sépare : Mounin, lui aussi souvent confronté à des textes énigmatiques, n'a jamais renoncé à la référence au vécu de l'auteur (bien qu'elle soit souvent difficile, parfois impossible, à retracer), ni à voir dans la poésie une communication véritable, et dans tout poème un message à tirer au clair. Pergnier, au contraire, plus proche en cela des vues d'un Michael Riffaterre, privilégie la référence au langage, toujours déchiffrable en théorie, puisqu'inscrite dans le texte. Et le traductologue qu'il est ne manque pas d'ajouter (à la même page) : " Tout traducteur sait bien que la traduction poétique laisse un goût d'insatisfaction, né de l'impossibilité de rendre dans le même nombre de mots la multiplicité des sens portés par le vers - voire de choisir parmi ces sens lequel est primordial, lequel secondaire ou contingent... »

Pour mener à bien ce qu'il appelle " une exploration de l'esthétique de l'équivoque » (p. 10), Pergnier choisit ses objets d'étude avec grand soin et les examine dans un ordre qui n'est pas indifférent, allant du moins au plus sémantiquement " piégé ». Le premier est un 
vers fort connu de Charles Baudelaire, Mais le vert paradis des amours enfantines, étudié dans son contexte. Cet énoncé n'a certes rien d'énigmatique, mais le choix et la place de la première épithète invitent déjà à une réflexion sur la polysémie - « Non pas le simple jeu des "connotations", précise-t-il, mais la plurivocité inhérente au signe " (p. 37). Les deux objets suivants sont des poèmes en trompe-l'œil de Cocteau, tirés de son recueil Opéra et intitulés L'Hôtel et BlasonOracle. Ils sont d'un bout à l'autre fabriqués d'énoncés équivoques : La mer veille. Le coq dort. La rue meurt de la mer... Le mot ment Ève nue! «En érigeant l'équivoque au rang de principe esthétique, [Cocteau] interroge ici non seulement la poésie, mais la langue, dans ses racines. À travers des jeux homonymiques complexes, c'est la relation même de la forme linguistique au sens, et l'arbitraire du signe, qui sont questionnés » (p. 56). Le dernier objet choisi est un tableau de René Magritte, La Grande Famille, qui, à l'instar des poèmes de Cocteau, est construit sur une équivoque " pren[ant] la consistance de l'énigme : l'analyse consiste donc essentiellement à "dévoiler» des sens que la facture de l'œuvre dissimule. La juxtaposition des analyses suffit à suggérer la parenté entre ce que le peintre crée avec des images et le poète avec des mots " (p. 10).

"Les objets esthétiques », premiers mots du livre de Pergnier, est un syntagme bien commode pour désigner à la fois un tableau et des poèmes. Mais surtout, il impose d'entrée de jeu la notion de matérialité de l'œuvre, entendue au sens de Gilles-Gaston Granger comme " un produit susceptible de se maintenir dans l'existence concrète et de s'offrir à l'observation, voire à l'usage, d'autres sujets que son créateur » (L'Irrationnel, Paris, Odile Jacob, 1998, p. 10).

Par ailleurs, le choix judicieux d'objets énigmatiques permet à Pergnier d'observer sous un fort grossissement (l'adjectif " paroxystique ", attribué au " travail de la pâte verbale ", revient plusieurs fois sous sa plume) la polysémie à l'œuvre dans le discours poétique (ou, métaphoriquement, pictural) - et par là, d'isoler un procédé d'écriture jusqu'ici mal $v u$, dont la prise en compte est de nature à permettre une lecture plus participante. Mais les observations de l'auteur ne s'arrêtent pas à ce stade heuristique, et les quatre analyses qu'il propose se terminent toutes par des interprétations ramenant le lecteur de l'étrangeté du poème, ou du tableau, au rationnel de son appréciation. Le vers de Baudelaire, par exemple, s'inscrit dans 
la structure « résolument dialogique [d'un] monologue dans lequel le poète prend à témoin [...] une femme répondant au beau nom d'Agathe " (p. 36); L'Hôtel énigmatique de Cocteau donne lieu à de multiples interprétations référentielles - une évocation de l'Hôtel Welcome à Villefranche, où ont été écrits la plupart des poèmes d'Opéra; " une séquence de brefs récits "; la frustration du désir sexuel (Le coq dort ; angl. cock, " pénis 》)... De même, le tableau de Magritte est une représentation cryptée de la Sainte Trinité (d'où le masque du titre : La Grande Famille).

Les poèmes et les tableaux ne sont évidemment pas toujours énigmatiques et ne se prêtent donc pas toujours à la lecture syncrétique de Pergnier - qui, sans doute, ne l'ignore pas. En effet, à côté des Illuminations d'Arthur Rimbaud, où le recours au " dérèglement de tous les sens " entraîne l'abandon de l'usage ordinaire du langage et crée des O.P.N.I. (P pour " poétiques ») qui ouvrent sur des mondes inconnus, tout constellés de sens, il y a Le Dormeur $d u$ val, du même Rimbaud. Ce poème pourra, certes, susciter des lectures "plurielles » (le soldat comme figuration du Christ?). Mais, par un engrenage textuel implacable, il contraint le lecteur à une interprétation manifeste, l'entrainant, à travers une nature luxuriante où s'accumulent d'inquiétants indices, jusqu'à la macabre découverte qui lui arrache, en point d'orgue, un cri d'effroi : " Il a deux trous rouges au côté droit! » Libre, ensuite, à tout lecteur de phantasmer à son gré sur la scène imposée.

Que dire, pour finir - d'une étude qu'on a trouvée stimulante à l'extrême, rigoureuse et pleine de finesse - d'autre que... sa lecture s'impose d'extrême urgence?

\author{
Claude Tatilon \\ Université York, Collège Glendon
}

